\title{
Combining independent de novo assemblies optimizes the coding transcriptome for nonconventional model eukaryotic organisms
}

\author{
Nicolas Cerveau and Daniel J. Jackson ${ }^{*}$
}

\begin{abstract}
Background: Next-generation sequencing (NGS) technologies are arguably the most revolutionary technical development to join the list of tools available to molecular biologists since PCR. For researchers working with nonconventional model organisms one major problem with the currently dominant NGS platform (Illumina) stems from the obligatory fragmentation of nucleic acid material that occurs prior to sequencing during library preparation. This step creates a significant bioinformatic challenge for accurate de novo assembly of novel transcriptome data. This challenge becomes apparent when a variety of modern assembly tools (of which there is no shortage) are applied to the same raw NGS dataset. With the same assembly parameters these tools can generate markedly different assembly outputs.
\end{abstract}

Results: In this study we present an approach that generates an optimized consensus de novo assembly of eukaryotic coding transcriptomes. This approach does not represent a new assembler, rather it combines the outputs of a variety of established assembly packages, and removes redundancy via a series of clustering steps. We test and validate our approach using Illumina datasets from six phylogenetically diverse eukaryotes (three metazoans, two plants and a yeast) and two simulated datasets derived from metazoan reference genome annotations. All of these datasets were assembled using three currently popular assembly packages (CLC, Trinity and IDBA-tran). In addition, we experimentally demonstrate that transcripts unique to one particular assembly package are likely to be bioinformatic artefacts. For all eight datasets our pipeline generates more concise transcriptomes that in fact possess more unique annotatable protein domains than any of the three individual assemblers we employed. Another measure of assembly completeness (using the purpose built BUSCO databases) also confirmed that our approach yields more information.

Conclusions: Our approach yields coding transcriptome assemblies that are more likely to be closer to biological reality than any of the three individual assembly packages we investigated. This approach (freely available as a simple perl script) will be of use to researchers working with species for which there is little or no reference data against which the assembly of a transcriptome can be performed.

Keywords: Transcriptome, De novo assembly, Eukaryote, Protein coding, Merge, Redundant

\footnotetext{
* Correspondence: djackso@gwdg.de

Department of Geobiology, Goldschmidtstr.3, Georg-August University of Göttingen, 37077 Göttingen, Germany
} 


\section{Background}

RNA-Seq is a flavour of NGS that can generate extremely powerful datasets for a variety of research themes. Gene discovery, digital gene expression profiling of entire tissues or developmental stages and population genetics [1,2] are some of the broad applications to which this technology can be applied. For researchers working with nonconventional model organisms RNASeq is alluring because such analyses are often touted as being possible in the absence of an assembled genome to which such transcriptome data is ideally mapped. In these cases the researcher faces the significant bioinformatic challenge of accurately assembling an RNA-Seq dataset "de novo" [3]. This is a challenge because the currently dominant NGS platform (Illumina) requires nucleic acid samples to be fragmented prior to sequencing, a process that needs to be accurately bioinformatically reversed in order to reconstruct the original transcripts. Additionally, typical Illumina read lengths are much less than 500 bp long [4]. These features result in both genome guided and de novo transcriptome assembly approaches displaying a large number of bioinformatically derived artefacts, a phemonenon that is well known [3]. The challenge of generating an accurate assembly of a transcriptome has generated many responses from the scientific community [5-9], with each assembly package having its own strengths and weaknesses. One de novo assembly strategy has been to generate multiple assemblies with different $\mathrm{k}$-mer values, to combine these and then remove the redundancy of the resulting merged assembly [10]. However this approach first requires the user to identify an appropriate range of k-mer values (not a trivial exercise), and may ultimately require the production of up to 62 transcriptomes for a single dataset [10]. Related to this issue is assessment of assembly quality. This issue is highlighted when one considers that different assembly packages applied to the same raw dataset usually generate markedly different outputs, even with the same assembly parameters, [10-12]. Any critical user would ask "which assembly is appropriate for my project?". For datasets with high proportions of "novel" genes (often the case for nonconventional model organisms), this problem has few solutions that can be generally applied to all datasets. Statistics such as the N50, average transcript size or coverage are not usually informative nor relevant when assessing the quality of an RNA-Seq assembly [13]. Another approach is to focus on the annotatability of a given assembly. In combination with standard sequence similarity searches against public databases, the recently released BUSCO (Benchmarking Universal Single-Copy Orthologs) package falls under this umbrella, and can be used to assess the completeness of a given transcriptome or genome assembly [14].
Having been through the process of de novo transcriptome assembly optimization with our nonconventional model Lymnaea stagnalis (a freshwater pulmonate mollusc), we have developed a simple strategy that takes the consensus coding features of a set of three (or more) independent assembly packages, and discards redundancy. This is not a new assembly method, but a way to survey the outputs of different assembly packages in order to generate a transcritpome that aims to be closer to the biological truth. We test our approach on simulated reads derived from the reference genomes of a fly and a nematode, and also on previously analyzed and publicly available raw RNA-Seq data derived from four eukaryotic lineages: two plants, a yeast, a fly and a nematode. In addition, we analyzed new RNA-Seq data derived from our model organism, Lymnaea stagnalis. For each dataset, we performed de novo assemblies with three independently developed and widely used software packages (Trinity [15], CLC Genomics Workbench V8.5 and IDBA-tran V1.1.1 [16]). The outputs of these assemblies were then processed through our pipeline. We demonstrate both bioinformatically (using a range of annotation based comparisons) and by validation in the lab for the L. stagnalis data, that this approach does indeed capture the most 'biologically correct' set of transcripts.

\section{Methods}

\section{Raw data acquisition}

We used Illumina NGS data previously reported from three well-established model organisms: Drosophila simulans, Caenorhabditis $s p$ and the unicellular eukaryote Saccharomyces cerevisiae. To increase the phylogenetic diversity of this selection we also included two plants, Hippophae rhamnoides and Nicotiana benthamiana. We also sampled the foot tissue of an individual Lymnaea stagnalis from our lab culture, and extracted total RNA following the protocol described in [17]. A stranded Truseq polyA library was constructed and paired end sequencing was performed on the Illumina HiSeq2000 platform. 46.5 million pairs raw reads were generated. 42.3 millon of these passed trimming and quality filtering and were used in all subsequent assembly analyses (Additional file 1: Table S1). The raw RNA-seq data for Drosophila simulans, Caenorhabditis sp., Saccharomyces cerevisiae, Hippophae rhamnoides and Nicotiana benthamiana were obtained from the NCBI sequence read archive (SRA) (respective accession numbers: SRR1956911, ERR690851, SRR1924287, SRP011938 and SRA066161 (single end data omitted). The FASTQ data files were extracted using the SRA tool kit provided by NCBI. For all datasets, individual reads were quality filtered using Trimmomatic V0.32 [18] with the following parameters: LEADING:5 TRAILING:5 MINLEN:36 (step 2 in Fig. 1). For L. stagnalis, TruSeq 


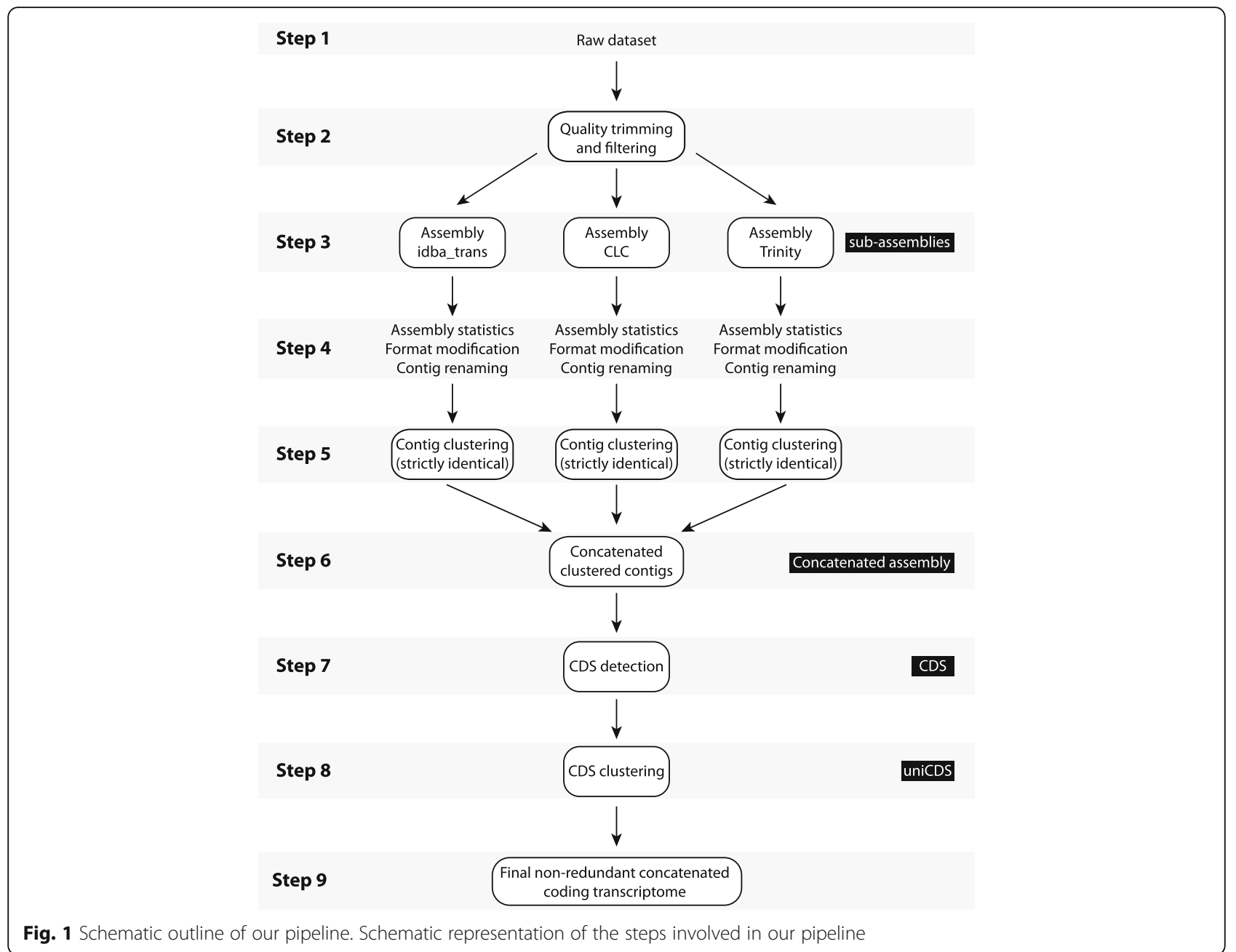

primer sequences were clipped with the following parameter: ILLUMINACLIP:primer_file:2:30:10. The five datasets used in this study contained between $46,166,144$ and 230,477,122 pairs of Illumina RNA-seq reads with read lengths of $100 \mathrm{bp}$, except for S. cerevisiae which had read lengths of $50 \mathrm{bp}$ (Additional file 1: Table S1). Between 83 and 100\% of the read pairs passed Trimmomatic quality checks (Additional file 1: Table S1). These quality filtered reads were used for our analyses.

\section{Generation of simulated Illumina reads from genomic references}

We also generated artificial reads derived from the reference genomes of two well-established model organisms: Drosophila melanogaster and Caenorhabditis elegans. Genomic reference sequences and gff annotations were donwloaded from NCBI database for D. melanogaster (GCF_000001215.4_Release_6_plus_ISO1_MT) and C. elegans (GCF_000002985.6_WBcel235). Gff annotations were transformed into gtf format using the 'rsem-gff3- to-gtf' command from the Rsem package with the option mRNA for the RNA-pattern parameter [19]. Some annotations had to be deleted because strand information was not consistent with other records of the same transcript or CDS. The gtf files contained 30,421 transcripts for D. melanogaster and 28,014 for C. elegans. The D. melanogaster genome is composed of 1870 sequences and the $C$. elegans genome is composed of 7 sequences. Transcripts were extracted from $D$. melanogaster and $C$. elegans genomes using 'rsem-prepare-reference' from the Rsem package with the options mRNA for gff3-RNApatterns and RefSeq for trusted-sources. Fifty Million read pairs were generated using the Flux simulator complete pipeline with simulated expression [20]. Library construction and sequencing simulation parameters for D. melanogaster are provided in Additional file 2. These artificially generated reads were also analyzed to calculate the read density per transcript. In order to represent variation in gene expression levels Flux simulator does not simulate reads on all input transcripts. We therefore removed transcripts that lacked simulated 
reads for all downstream analyses. As for Illumina datasets, simulated reads were quality filtered using Trimmomatic V0.32 [18] with the following parameters: LEADING:5 TRAILING:5 MINLEN:36 (step 2 in Fig. 1). In both datasets, $99.4 \%$ of the read pairs passed Trimmomatic quality checks (Additional file 1: Table S1). These reads were used for our analyses.

\section{Transcriptome assemblies}

We selected three assembly packages with unique assembly strategies for our investigation: Trinity V2.0.3 [15], CLC Genomics Workbench V8.5 and IDBA-tran [16]. While all of these packages employ the De Bruijn method to perform their assemblies, CLC and Trinity use a single k-mer method whereas IDBA-tran uses a multiple k-mer method. In addition, while CLC and IDBA-tran produce a single De Bruijn graph (per k-mer for IDBA-tran and for the whole dataset for CLC), Trinity produces one De Bruijn graph per transcript, which are subsequently processed independently in order to extract all splice isoforms and to separate paralogous genes.

For each of the eight datasets we performed one assembly with each assembly package, resulting in three independent assemblies per dataset (step 3 in Fig. 1). CLC assemblies were run using a word of 20 base pairs (bp), a bubble size of $50 \mathrm{bp}$, with reads mapped back to the transcriptome using default parameters. IDBA_tran assemblies were run with $\mathrm{k}$-mer values ranging from 20 to $100 \mathrm{bp}$ with a step size of $10 \mathrm{bp}$. Trinity assemblies were run with default parameters and a k-mer value of 25. For each of these independent assemblies we recorded a variety of statistics (number of transcripts, smallest transcript, largest transcript, median transcript size, total assembly size, N50; step 4 in Fig. 1).

\section{Concatenated-assembly generation}

Once the three individual assemblies for a given dataset had been generated we next produced a concatenated assembly. To do this we harmonized all assembly outputs into the same format. Transcript names were also modified so that the origin of each sequence in the concatenated-assembly could be traced (step 4 in Fig. 1). We then performed an intra-assembly clustering step in order to remove all strictly redundant transcripts present within each of the individual sub-assemblies for each dataset (step 5 in Fig. 1). For this clustering step we used CD-HIT-EST [21] with ten threads (-T), a maximum memory of 2549 megabytes $(-\mathrm{M})$, local sequence identity (-G 0) with identity parameter of $100 \%$ (-c 1.00), minimal coverage ratio of the shorter sequence of $100 \%(-\mathrm{aS}$ 1.00 ) and minimal coverage ratio of the longest sequence of $0.005 \%$ ( $-\mathrm{aL} 0.005)$. The minimal ratio of the longest sequence was chosen in order to allow clustering of the whole range of transcript sizes. The resulting unique transcripts derived from each of the 3 assemblies for each dataset were then concatenated (step 6 in Fig. 1). TransDecoder V2.0.1 [22] was then used to detect open reading frames (ORFs) greater than 100 amino acids (step 7 in Fig. 1). The resulting coding sequence (CDS i.e. with 5' and 3' UTRs removed), were then clustered again using CD-HIT-EST with minimal coverage ratio of the longest sequence of $0.005 \%$ (-aL 0.005), but a slightly lower sequence identity than the previous clustering step (-c 0.98) in order to take in consideration the Illumina sequencing error rate (step 8 in Fig. 1). The only parameter that can vary between clustering runs at this stage was the minimal coverage ratio of the shorter sequence $(-\mathrm{aS})$. This parameter had values that ranged from 75 to $100 \%(100,99,98,97,96,95,90,85,80$ and $75 \%)$. An -aS value was retrospectively selected in order to generate the most concise assembly (see below under in silico testing of assemblies). The resulting cluster info file (".clstr) was retained in order to identify the transcript that generated the longest CDS of each cluster, and also all other transcripts of this cluster for further analyses. We mined the cluster information file to determine the assembly origins of each CDS and to calculate the CDS extension size (see below). The consensus of each cluster was then classified into one of seven categories:

1. The cluster consensus was present in all three assembler outputs

2. The cluster consensus was present in CLC and IDBA_tran outputs

3. The cluster consensus was present in CLC and Trinity outputs

4. The cluster consensus was present IDBA_tran and Trinity outputs

5. The cluster consensus was present only present in the CLC output

6. The cluster consensus was present only present in the IDBA_tran output

7. The cluster consensus was present only present in the Trinity output

The perl script (concatenator.pl) used to perform all of these steps is provided in the Additional file 3. As input this script requires the path to a directory containing the assembly outputs to concatenate, and the paths to two binary files: CD-HIT-EST and TransDecoder. Variable options include the nucleotide identity and the minimal coverage ratio of the shortest sequence for the CDS clustering step (step 8 in Fig. 1).

\section{Transcriptome assembly quality control}

In order to test the quality of the assemblies generated by our pipeline we adopted two approaches, an 
annotatability based approach (applied to all datasets), and in vitro validation (applied to our L. stagnalis dataset).

\section{Annotatability of assemblies}

These analyses were performed with two different goals in mind. The first was to retrospectively determine the best minimal coverage ratio (aS value) for the final clustering step (in order to minimize redundancy and loss of information). To this end, we performed BLASTx searches for each of the above listed aS values, and BUSCO analyses for all assemblies based on Illumina datasets [14]. For BLASTx searches the e-value was set to $1 \mathrm{e}-3$. A perl script was used to count the number of CDSs with a BLASTx hit. In addition, the number of unique BLASTx hits were counted. These values were compared across the different assemblies in order to identify at which aS value the concatenated-transcriptome began to lose information. The second goal was to evaluate any improvement that our concatenated-assembly approach gave relative to each of the individual assemblers. We applied Transdecoder to the transcripts generated by each individual assembler with the same parameters as described above. Subsequent BLASTx searches were also performed as described above for the concatenated-assembly. In addition, BUSCO analyses of individual and concatenated transcriptomes were also compared.

\section{in vitro validation of the L. stagnalis assemblies}

We performed a small scale in vitro validation of our new L. stagnalis transcriptome data using 10 randomly selected transcripts from each of the following categories outlined above: 1, 5, 6 and 7. Although this is a small sample compared to the overall transcriptome size, the resulting trends were informative. Transcripts were selected randomly using a perl script (Additional file 4). We designed primer pairs for each of these 40 selected transcripts with a melting temperature of $60{ }^{\circ} \mathrm{C}$ using Primer3 [23]. RT-PCR was performed on foot total RNA isolated from three L. stagnalis individuals (RNA derived from the individual used for NGS sequencing was not used in this exercise). Reverse transcription reactions were performed in a final volume of $25 \mu \mathrm{L}$ as follows. One microgram of high quality total RNA was combined with $200 \mu \mathrm{mols}$ of random hexamer and water to a final volume of $10 \mu \mathrm{L}$. This mix was put at $70{ }^{\circ} \mathrm{C}$ for $5 \mathrm{~min}$ in order to melt RNA secondary structure and allow primer annealing. The mix was then cooled to room temperature. We then added to each reaction Promega 5X MMLV-RT buffer (final concentration 1X), dNTPs (final concentration of $0.4 \mathrm{mM}$ ), 200 Units of MMLV$\mathrm{RT} \mathrm{H}^{-}$mutant (Promega), and water to a final volume of $25 \mu \mathrm{L}$. For each reaction we performed a positive reverse transcription $(\mathrm{RT}+)$ containing all components mentioned above, and a negative reaction where MMLV-RT was replaced by water (RT-) to control for contaminating genomic DNA. Both RT+ and RT- reactions were incubated at room temperature for $10 \mathrm{~min}$, and then heated to $42{ }^{\circ} \mathrm{C}$ for $90 \mathrm{~min}$. The reactions were then heated to $70{ }^{\circ} \mathrm{C}$ for $15 \mathrm{~min}$ to inactivate the MMLV-RT. Single stranded cDNA was stored at $-20^{\circ} \mathrm{C}$. PCR reactions were performed in a final volume of $25 \mu \mathrm{L}$ containing the following: a final concentration of $1 \mathrm{X}$ enzyme reaction buffer, $0.2 \mathrm{mM}$ dNTPs, $0.2 \mu \mathrm{M}$ forward and reverse primers, $0.5 \mathrm{U}$ Q5 polymerase (NEB), $1 \mu \mathrm{L}$ of cDNA template and water to a final volume of $25 \mu \mathrm{L}$. Thermocycling was were performed in a SensoQuest thermocycler with the following steps: $94{ }^{\circ} \mathrm{C}$ for $10 \mathrm{~min}, 35$ cycles with denaturation at $94{ }^{\circ} \mathrm{C}$ for $30 \mathrm{~s}$, primer annealing at $55{ }^{\circ} \mathrm{C}$ for $30 \mathrm{~s}$, DNA synthesis at $72{ }^{\circ} \mathrm{C}$ for 3 min with a final elongation step at $72{ }^{\circ} \mathrm{C}$ for $10 \mathrm{~min}$. PCR products were loaded onto a $2 \%$ agarose gel containing ethidium bromide and electrophoresed at $130 \mathrm{~V}$ for 40 to $50 \mathrm{~min}$ and then visualized under UV light. For each primer pair, results were considered congruent when all three replicate $\mathrm{RT}+$ reactions contained a distinct band at the expected size, and all three replicate RT- reactions were negative. A result was considered incongruent in any other case for the RT+ reactions. Reactions with negative and incongruent results were repeated a second time to confirm the results.

\section{Results and discussion}

\section{Individual transcriptome assemblies}

In order to broadly compare the outputs of the individual assemblers (CLC, Trinity and IDBA_tran) with our concatenated assemblies, we calculated some standard assembly metrics that are commonly used to characterize these kinds of datasets [13]. While each assembly output displayed different characteristics, a consistent pattern could be observed. Assemblies produced by Trinity always produced the highest numbers of transcripts and the largest transcriptome sizes (as measured by cumulating transcript lengths), whereas CLC generated assemblies with the lowest numbers of transcripts (except for the S. cerevisiae and C. elegans samples), and the smallest transcriptome sizes (Additional file 5: Table S2). Number of input reads did not have any influence on these metrics. Indeed, S. cerevisiae dataset has approximately twice the number of input reads than all other samples, and the smallest transcriptome output regardless of the assembly software. In general CLC and IDBA_tran produced 2.2 to 4.6 and 1.8 to 3.6 times fewer transcripts than Trinity respectively. N50 values for all assemblers lay between 405 and 4056 bp. IDBA_tran consistently generated the longest N50s, and CLC generally the smallest (except for the $S$. cerevisiae, $H$. rhamnoides and C. elegans datasets; Additional file 5: 
Table S2). However we must point out that the number of transcripts and N50 values will be biased by differences in the smallest transcript size assembled by each software (300 bp for IDBA-tran, 211 for CLC and 201 for Trinity; Additional file 5: Table S2), and also by the biological realities of these transcriptomes - longer N50 values do not necessarily reflect a better transcriptome assembly. The longest transcript sizes varied from 8609 to $51,362 \mathrm{bp}$, with the longest transcripts generated by Trinity (except for the $H$. rhamnoides, $N$. benthamiana, D. melanogaster and C. elegans datasets where it was generated by IDBAtran). Interestingly, for some datasets the longest transcript size varied by more than two fold according to the assembler used (Additional file 5: Table S2). These general observations confirm previous reports that the use of different assemblers (even though they are all based on the construction of de Bruijn graphs), generate significantly different final assemblies [12, 24-26]. This led us to explore the possibility of combining these assemblies and removing any redundancy.

\section{Concatenated transcriptome assemblies}

The main goal of our concatenated assembly approach was to improve assembly accuracy without generating a bloated assembly. In order to first remove intraassembly redundancy, a stringent clustering step (100\% sequence identity on $100 \%$ of the shorter sequence length) was performed for each individual sub-assembly (step 5 in Fig. 1). For all datasets, the redundancy rate was zero for all IDBA_tran assemblies and below 0.02\% for all CLC assemblies (Additional file 6: Table S3). For Trinity transcriptomes, the redundancy rate was always significantly higher and ranged between 0.02 and 30\% (Additional file 6: Table S3). The redundancy in the Trinity assemblies was also higher in the two simulated datasets (27 and 30\%) than in the Illumina datasets (maximum 11\%) (Additional file 6: Table S3). Higher intra-Trinity redundancy is probably due to the fact that Trinity is the only assembler to produce one de Bruijn graph per transcript, and subsequently processes them one by one, whereas CLC and IDBA_trans produce only one graph overall. The non-redundant transcripts produced by each assembler for each dataset were then pooled and TransDecoder was used to detect putative ORFs with a size of $\geq 100$ amino acids. The resulting datasets had concatenated transcriptomes with 25,854 to 885,944 transcripts, and TransDecoder detected between 22,180 and 379,596 putative ORFs (Additional file 7: Table S4). Both simulated datasets fell within the range described by the Illumina datasets, while the proportion of the simulated transcriptomes predicted to be coding was higher (Additional file 7: Table S4). This is most probably due to the fact that simulated reads were derived from mRNA molecules.
The next critical step was to cluster the CDSs produced by Transdecoder in order to obtain the most concise coding transcriptome while minimizing information loss (step 8 in Fig. 1). To do this we used CD-HIT-EST with the nucleotide identity level set to $98 \%$ in order to be more conservative than the average Illumina sequencing error rate of $1 \%$. The size ratio of the longest transcript to the overall transcript was set to $0.5 \%$ in order to include the shortest transcripts. The size ratio of the shortest transcript to the overall transcript $(-\mathrm{aS})$ varied from 100 to $75 \%$ (see below). To evaluate the amount of information lost at this step, we used annotation-based metrics [13] that make more biological sense than metrics such as N50 or transcript size (however these can be found in Additional file 5: Table S2). BLASTx searches against Swiss-Prot database for each $\mathrm{aS}$ value were performed to determine the impact of the aS value in the above clustering step. It showed that the number of unique database entries decrease at $99 \%$ for both Illumina derived and simulated datasets (Additional file 8: Table S5). In addition, BUSCO analyses also showed that the completeness of each assembly began to decrease at an aS value of $99 \%$ for each sample. On the basis of these analyses (and to be the most conservative), the smallest aS value was set to $100 \%$ for all datasets. Nevertheless, it should be kept in mind that according to the dataset and the type of downstream analysis to be performed, a lower aS value may be more appropriate. After this clustering exercise, between 54 and $68 \%$ of the CDSs from each dataset were found to be redundant at the nucleotide level (Table 1). C. elegans dataset is in the range of the Illumina datasets whereas D. melanogaster is two point higher than the highest Illumina dataset, which is $D$. simulans.

Our concatenated coding transcriptomes ranged in size from 9744 transcripts for S. cerevisiae to 127,526 transcripts for $N$. benthamiana (Table 1). Whatever the raw data origin, the number of transcripts in the concatenated assembly is less than the number of uniCDSs, and the number of CDSs in the concatenated assembly is more than the number of uniCDSs (Table 1). This is because transcripts often possessed more than one CDS (Table 1). Transcripts with multiple CDSs also influenced the number of redundant CDSs in our concatenated assemblies (between 3 and 22\%, Table 1). The proportion of CDS redundancy for the simulated $D$. melanogaster data is within the range of all Illumina datasets, while the simulated C. elegans is $5 \%$ lower than the smallest Illumina dataset ( $H$. rhamnoides $8 \%$ ). Transcripts with multiple CDSs may be the result of sequencing or assembly errors, the activity of transposable elements such as group-II intron or transposases that get inserted in genes [27], or operon transcription [28]. Compared to the individual assemblies generated 
Table 1 Assembly statistics

\begin{tabular}{|c|c|c|c|c|c|c|c|c|c|}
\hline & \multirow[b]{2}{*}{ Step ${ }^{a}$} & \multicolumn{6}{|c|}{ Illumina derived datasets } & \multicolumn{2}{|c|}{ Simulated datasets } \\
\hline & & L. stagnalis & S. cerevisiae & Caenorhabditis $s p$. & D. simulans & H. rhamnoides & N. benthamiana & D. melanogaster & C. elegans \\
\hline $\begin{array}{l}\text { Number of concatenated } \\
\text { transcripts }\end{array}$ & 6 & 576,412 & 25,854 & 152,491 & 184,892 & 278,987 & 885,944 & 42,535 & 15,340 \\
\hline CDS number & 7 & 139,727 & 22,180 & 112,813 & 81,598 & 137,601 & 379,596 & 37,920 & 41,103 \\
\hline uniCDS number ${ }^{b}$ & 8 & $\begin{array}{l}59,178 \\
(58 \%)\end{array}$ & $\begin{array}{l}9,942 \\
(55 \%)\end{array}$ & $\begin{array}{l}40,116 \\
(64 \%)\end{array}$ & $\begin{array}{l}27,735 \\
(66 \%)\end{array}$ & $\begin{array}{l}63,092 \\
(54 \%)\end{array}$ & $\begin{array}{l}131,656 \\
(65 \%)\end{array}$ & $\begin{array}{l}12,118 \\
(68 \%)\end{array}$ & $\begin{array}{l}14,890 \\
(64 \%)\end{array}$ \\
\hline Total transcript number & 9 & 58,185 & 9,744 & 39,022 & 26,968 & 61,798 & 127,526 & 11,582 & 14,283 \\
\hline Total CDS number & 9 & 64,659 & 11,605 & 51,416 & 34,363 & 68,288 & 153,118 & 14,231 & 15,412 \\
\hline $\begin{array}{l}\text { Transcripts with multiple } \\
\text { CDSs }^{c}\end{array}$ & 9 & $\begin{array}{l}5,759 \\
(10 \%)\end{array}$ & $\begin{array}{l}1,529 \\
(15 \%)\end{array}$ & $\begin{array}{l}9,756 \\
(19 \%)\end{array}$ & $\begin{array}{l}5,838 \\
(22 \%)\end{array}$ & $\begin{array}{l}5,999 \\
(10 \%)\end{array}$ & $\begin{array}{l}21,060 \\
(17 \%)\end{array}$ & $\begin{array}{l}2,218 \\
(19 \%)\end{array}$ & $\begin{array}{l}949 \\
(7 \%)\end{array}$ \\
\hline Redundant $\mathrm{CDSs}^{\mathrm{d}}$ & 9 & $\begin{array}{l}5,481 \\
(9 \%)\end{array}$ & $\begin{array}{l}1,663 \\
(14 \%)\end{array}$ & $\begin{array}{l}11,300 \\
(22 \%)\end{array}$ & $\begin{array}{l}6,628 \\
(19 \%)\end{array}$ & $\begin{array}{l}5,196 \\
(8 \%)\end{array}$ & $\begin{array}{l}21,462 \\
(14 \%)\end{array}$ & $\begin{array}{l}2,113 \\
(15 \%)\end{array}$ & $\begin{array}{l}522 \\
(3 \%)\end{array}$ \\
\hline Transcriptome size (bp) & 9 & $131,591,076$ & $16,164,888$ & $69,689,679$ & $69,421,322$ & $86,181,833$ & $206,036,224$ & $34,121,269$ & $19,765,122$ \\
\hline Smallest transcript (bp) & 9 & 300 & 300 & 300 & 300 & 300 & 300 & 300 & 300 \\
\hline Largest transcript (bp) & 9 & 35,470 & 15,061 & 21,466 & 51,362 & 13,117 & 19,833 & 29,220 & 26,756 \\
\hline N50 & 9 & 3,483 & 2,414 & 2,366 & 3,866 & 1,823 & 2,116 & 4,479 & 1,666 \\
\hline
\end{tabular}

${ }^{\mathrm{a}}$ Step number in Fig. 1

${ }^{\text {b }}$ Proportion of discarded CDSs is indicated in brackets

'Proportion of transcripts with $>1$ CDS is indicated in brackets

${ }^{d}$ Proportion of none unique CDSs is indicated in brackets

by CLC, Trinity and IDBA-tran (Additional file 5: Table S2), the concatenated assemblies of $L$. stagnalis, D. simulans and $N$. benthamiana contained fewer transcripts than any of the individual sub-assemblies, whereas for $S$. cerevisiae, Caenorhabditis sp, H. rhamnoides, D. melanogaster and C. elegans the number of transcripts within the concatenated assemblies were within range of those produced by the individual assemblers. Considering the total transcriptome sizes, the concatenated assemblies were similar to the individual assemblies, but were always larger than the CLC generated assemblies (Table 1; Additional file 5: Table S2). For the S. cerevisiae dataset the concatenated transcriptome was larger than all individual assemblies (Table 1; Additional file 5: Table S2). Finally, the N50s of the concatenated assemblies were higher than all of the individual assemblies except for the S. cerevisiae and C. elegans dataset. This suggests that most of the transcripts removed during our concatenation and filtering steps had small sizes. These statistics also show that our pipeline did not increase the overall transcriptome size compared to the individual assemblers. In some cases the overall transcriptome size even decreased considerably (Table 1). This phenomena has also been previously observed in other plant datasets $[6,10]$. The N50 values also suggests that our pipeline generates coding transcriptomes that have larger average transcript sizes than assemblies generated by the individual assemblers [13].

In order to further assess the performance of our pipeline, we compared transcripts generated by the three individual assemblers and our pipeline with the original transcripts from which artificial reads were generated for both the $D$. melanogaster and $C$. elegans datasets (Table 2). These comparisons were performed with BLASTn and we only considered hits with a nucleotide identity of $98 \%$ covering at least $50 \%$ of the original transcript. In both datasets, CLC failed to recover the highest proportion of genuine transcripts $(21 \%$ for $D$. melanogaster and $34 \%$ for C. elegans), while our concatenated assemblies failed to recover the lowest proportion of genuine transcripts ( $11 \%$ for D. melanogaster and $29 \%$ for C. elegans). In general, these concerningly high values are similar to those previously made on human and worm de novo transcriptome assemblies [3]. For both the D. melanogaster and C. elegans datasets, most of the missing genuine transcripts in the concatenated assembly ( $85 \%$ for D. melanogaster and $80 \%$ for C. elegans) had read coverages of less than 10X, whereas most of the successfully recovered transcripts ( $82 \%$ for D. melanogaster and $89 \%$ for C. elegans) had read coverages higher than 10X.

Interestingly, and in contrast to the missing genuine transcripts, up to $27 \%$ of the assembled transcripts were not present in the original transcript set (representing bioinformatically 'invented' transcripts). IDBA_tran produces the lowest proportion of invented transcripts (14\% for D. melanogaster and $14 \%$ for C. elegans), whereas Trinity produces the highest proportion of invented transcripts in D. melanogaster (25\%) and CLC in C. elegans (25\%). In C. elegans, our concatenated assembly 
Table 2 Comparison between original and assembled transcriptomes derived from simulated reads generated from $D$. melanogaster and C. elegans datasets

\begin{tabular}{|c|c|c|c|c|c|}
\hline \multirow[t]{2}{*}{ Organism } & \multirow[t]{2}{*}{ Assembler } & \multicolumn{2}{|c|}{ Original transcripts } & \multicolumn{2}{|c|}{ Assembled transcripts } \\
\hline & & Total number & $\begin{array}{l}\text { Lacking a BLASTn hit in assembled } \\
\text { transcripts }\end{array}$ & Total number & $\begin{array}{l}\text { Lacking a BLASTn hit in original } \\
\text { transcripts }\end{array}$ \\
\hline \multirow[t]{4}{*}{ D. melanogaster } & Concatenated & 11,856 & $1,277(11 \%)$ & 12,273 & $2,685(22 \%)$ \\
\hline & CLC & & $2,513(21 \%)$ & 8,113 & $1,972(24 \%)$ \\
\hline & IDBA_tran & & $1,775(15 \%)$ & 8,282 & $1,150(14 \%)$ \\
\hline & Trinity & & $1,843(16 \%)$ & 11,395 & $2,810(25 \%)$ \\
\hline \multirow[t]{4}{*}{ C. elegans } & Concatenated & 16,513 & $4,774(29 \%)$ & 14,922 & $4,087(27 \%)$ \\
\hline & CLC & & $5,614(34 \%)$ & 11,853 & $2,948(25 \%)$ \\
\hline & IDBA_tran & & $5,018(30 \%)$ & 11,069 & $1,557(14 \%)$ \\
\hline & Trinity & & $5,473(33 \%)$ & 12,843 & $2,869(22 \%)$ \\
\hline
\end{tabular}

had a higher proportion of invented transcripts than any single assembler, whereas in D. melanogaster it had a lower proportion than CLC and Trinity (Table 2).

\section{Evaluation of concatenated assemblies}

In order to study the composition of the final uniCDS clusters in our concatenated assemblies we assigned all clusters to one of seven categories (Fig. 2). The resulting pattern was consistent across all datasets, and all aS ratios used (75-100\%) in the clustering step (data not showed). CDS clusters primarily belonged to either category 1 (the cluster was present in all three individual sub-assemblies following concatenation and redundancy filtering) or category 6 (the cluster was only present in the Trinity assembly) (Fig. 2). Of all three individual assemblers, Trinity consistently generated the most unique clusters (excepted for C. elegans), while CLC consistently generated the fewest unique clusters (excepted for $C$. elegans) (Fig. 2).

In order to compare these distributions between samples, we performed Kolmogorov-Smirnov statistical tests. All paired comparisons were statistically non-significant except for 4 that always involved at least one of the plant transcriptomes (one for $\mathrm{H}$. rhamnoides and three for $N$. bentamiana) (Additional file 9: Table S6). Distribution comparisons between concatenated assemblies from both simulated datasets without plant Illumina datasets were always non-significant (Additional file 9: Table S6).

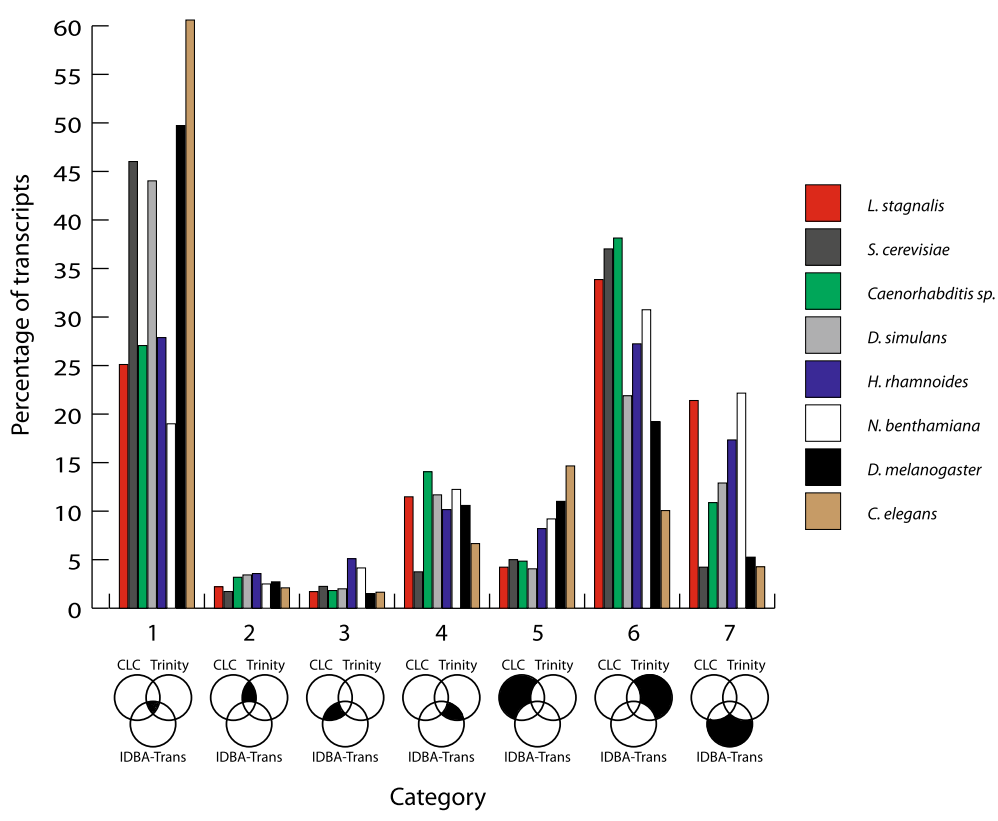

Fig. 2 Categorization of concatenated clusters according to their presence/absence in the individual sub-assemblies. Category 1: clusters found in all three assemblers; category 2: clusters found in CLC and Trinity; category 3: clusters found in CLC and IDBA; category 4: clusters found in IDBA-tran and Trinity; category 5: clusters found in CLC; category 6: clusters found in Trinity and category 7: clusters found in IDBA 
This categorization exercise led us to ask whether any one of these categories contained a higher proportion of "biologically correct" transcripts than others? In order to address this question, we performed an in vitro validation using the $L$. stagnalis dataset. We tested ten randomly selected clusters from categories 1 (clusters detected in all three assemblers), 5, 6 and 7 (clusters unique to either CLC, Trinity or IDBA_tran respectively). The positive validation rate for categories 5, 6 and 7 ranged from 40 to $70 \%$, and the negative validation rate ranged from 30 to $60 \%$ (Table 3 ). Category 1 had a positive validation rate of $80 \%$, and a negative validation rate of $0 \%$ (Table 3 ). These results suggest that clusters found by only one assembler (categories 5, 6 or 7) are likely to be either very lowly expressed or are assembly errors, while those found in all three assemblers (category 1) are more likely to be genuine molecules, giving further credence to the concept of our bioinformatic approach.

We also retrospectively investigated the completeness of each individual assembly relative to our concatenated assemblies. The results of this analysis were striking. Averaging across all eight datasets, $50.3 \% \pm 16.1 \%$ of CDS clusters in the concatenated assembly were present in the CLC assemblies, $62.3 \% \pm 7.1 \%$ were present in the IDBA_tran assemblies and $77.5 \% \pm 8.0 \%$ were present in the Trinity assemblies (Fig. 3a). Both simulated concatenated assemblies were in the range of all Illumina derived assemblies, excepted for CLC where the proportion of detected CDSs was higher than in any of the 6 other samples (Fig. 3a). On face value this result suggests that Trinity alone provides the best overall picture of a coding transcriptome. However, when we looked retrospectively at the effect of our pipeline on CDS extension, we found IDBA_tran to be the best performer for all datasets (except for both Caenorhabditis datasets; Fig. 3b). Between 13 and 44\% of the CDSs from each assembler were extended during our concatenating process (Table 4). The proportion of extended CDSs from the simulated transcriptomes were within the range

Table $\mathbf{3}$ In vitro validation of L. stagnalis clusters

\begin{tabular}{lllll}
\hline & Number & Positive & Incongruent & Negative \\
\hline $\begin{array}{l}\text { Category 1 } \\
\text { Clusters found in all 3 }\end{array}$ & 10 & 8 & 2 & 0 \\
assemblers \\
$\begin{array}{l}\text { Category 5 } \\
\text { Clusters unique to the CLC } \\
\text { assembly }\end{array}$ & 10 & 2 & 5 & 3 \\
$\begin{array}{l}\text { Category 6 } \\
\text { Clusters unique to the }\end{array}$ & 10 & 2 & 2 & 6 \\
$\begin{array}{l}\text { Trinity assembly } \\
\text { Category 7 } \\
\text { Clusters unique to the }\end{array}$ & 10 & 4 & 1 & \\
IDBA_tran assembly & & & & \\
\hline
\end{tabular}

of all Illumina derived assemblies, excepted for CLC in C. elegans which was $3 \%$ lower than the smallest Illumina dataset (IDBA_tran in S. cerevisiae) (Table 4).

We also compared the annotatability of our concatenated transcriptomes relative to assemblies generated by each of the three individual assemblers using BLASTx sequence similarity searches against Swiss-Prot [13]. The results of these analyses showed that annotatability was always higher in the concatenated assemblies compared to all of the individual assemblies (Table 5). For all Illumina derived datasets, the proportion of CDSs with a BLASTx hit expressed as a percentage of that found in the corresponding concatenated assembly ranged between $94 \%$ for the Trinity assembly of the $S$. cerevisiae dataset to $36 \%$ for the CLC assembly of the $L$. stagnalis dataset (Table 5). This trend also held true for the $D$. melanogaster and $C$. elegans simulated datasets (Table 5).

We were aware that an increase in the proportion of CDSs returning a BLASTx hit does not necessary mean that annotation diversity also increases. Indeed, an overall increase in the number of BLASTx hits could be due to a greater number of mis-assembled isoforms or paralogs present in a given assembly. To account for this phenomenon we investigated annotation diversity by calculating the number of unique database entries for all BLASTx searches. Again in all cases the number of unique BLASTx hits was highest in the concatenated assemblies (Table 5). For the Illumina datasets, the number of unique database hits in the individual assemblies expressed as a percentage of that found in the corresponding concatenated assembly ranged between 98\% (for the Trinity assembly of the Caenorhabditis $s p$. dataset) and $72 \%$ (for the CLC assembly of the L. stagnalis dataset; Table 5). These results demonstrate that an overall increase in the rate of annotation is accompanied by an increase in annotation diversity. This phenomena was also observed in the analysis of a $N$. benthamiana transcriptome [10]. It should be noted that the increase in annotation diversity in our concatenated assemblies was less extreme than the increase in the overall annotatability (Table 5). This implies that most of the increase in the overall annotation is due to CDS isoforms that were not found by a given individual assembler.

We also performed an analysis of assembly completeness using the transcription factor database BUSCO [14]. In addition to the simple presence/absence pattern of BUSCO entries, this analysis also provides interesting information regarding the number of duplicated and fragmented entries. The results of this analysis also confirmed the results obtained with our BLASTx searches; the number of detected BUSCOs entries was always higher in the concatenated assemblies than in all of the individual assemblers for all Illumina datasets and the 


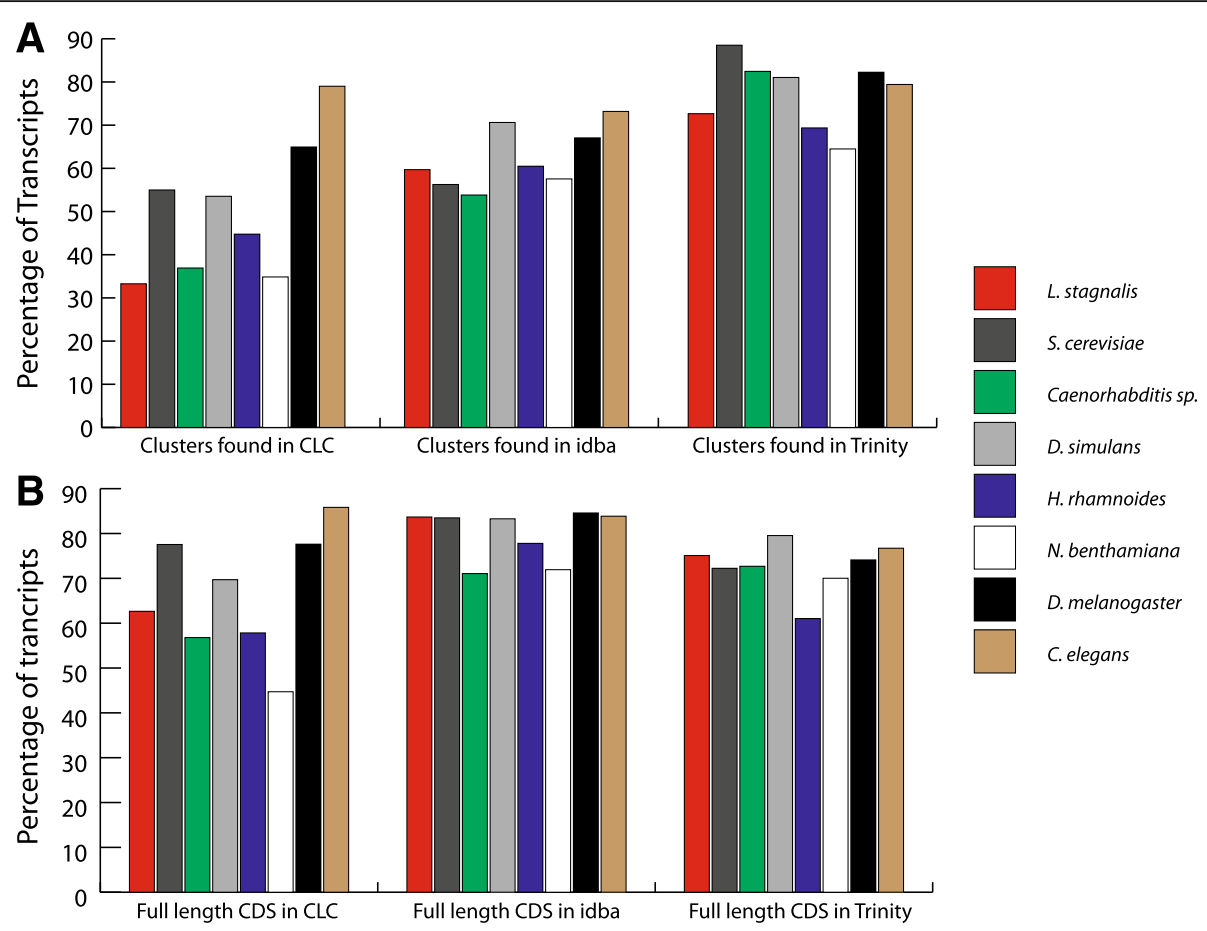

Fig. 3 Characterisation of CDSs present in the final concatenated assembly and their presence/absence in the individual sub-assemblies. a Proportion of CDSs present in the final concatenated assembly present in each individual assembler for each dataset. b For those CDSs present in each sub-assembler (as in A), the proportion of CDSs from each individual assembler that matches (or exceeds) the length of the final concatenated CDS

simulated datasets (Table 6). In addition, the number of fragmented copies was always lower in all concatenated assemblies than in the individual sub-assemblies, except for the Caenorhabditis sp. dataset where the number of fragmented copies was equal in the concatenated and IDBA_tran assemblies and the $C$. elegans dataset where the number of fragmented copies is lower in Trinity and equal in IDBA_tran (Table 6).

There were always the fewest number of duplicated copies in all CLC sub-assemblies, but CLC was always the single assembler with the fewest total number of BUSCO entries, except for the $S$. cerevisiae and $C$.

Table 4 Effect of concatenating assemblies on CDS length

\begin{tabular}{|c|c|c|c|c|c|c|c|c|c|}
\hline & \multirow[b]{2}{*}{ Assembler } & \multicolumn{6}{|c|}{ Illumina derived datasets } & \multicolumn{2}{|c|}{ Simulated datasets } \\
\hline & & L. stagnalis & S. cerevisiae & Caenorhabditis sp. & D. simulans & H. rhamnoides & N. benthamiaana & D. melanogaster & C.elegans \\
\hline \multirow[t]{3}{*}{$\begin{array}{l}\text { Number of extended } \\
\text { CDSs }^{\mathrm{a}}\end{array}$} & $\mathrm{CLC}$ & $\begin{array}{l}8,195 \\
(38 \%)\end{array}$ & $\begin{array}{l}1,333 \\
(23 \%)\end{array}$ & $\begin{array}{l}7,255 \\
(44 \%)\end{array}$ & $\begin{array}{l}5,490 \\
(34 \%)\end{array}$ & $\begin{array}{l}11,508 \\
(38 \%)\end{array}$ & $\begin{array}{l}24,218 \\
(47 \%)\end{array}$ & $\begin{array}{l}1,888 \\
(23 \%)\end{array}$ & $\begin{array}{l}1,534 \\
(13 \%)\end{array}$ \\
\hline & IDBA_tran & $\begin{array}{l}13,401 \\
(27 \%)\end{array}$ & $\begin{array}{l}957 \\
(16 \%)\end{array}$ & $\begin{array}{l}11,717 \\
(38 \%)\end{array}$ & $\begin{array}{l}7,586 \\
(25 \%)\end{array}$ & $\begin{array}{l}11,427 \\
(24 \%)\end{array}$ & $\begin{array}{l}48,830 \\
(36 \%)\end{array}$ & $\begin{array}{l}1,960 \\
(23 \%)\end{array}$ & $\begin{array}{l}2,054 \\
(18 \%)\end{array}$ \\
\hline & Trinity & $\begin{array}{l}23,532 \\
(34 \%)\end{array}$ & $\begin{array}{l}2,879 \\
(27 \%)\end{array}$ & $\begin{array}{l}28,918 \\
(44 \%)\end{array}$ & $\begin{array}{l}11,638 \\
(33 \%)\end{array}$ & $\begin{array}{l}21,032 \\
(35 \%)\end{array}$ & $\begin{array}{l}78,038 \\
(40 \%)\end{array}$ & $\begin{array}{l}7,817 \\
(38 \%)\end{array}$ & $\begin{array}{l}5,808 \\
(33 \%)\end{array}$ \\
\hline \multirow{3}{*}{$\begin{array}{l}\text { Cumulated extended } \\
\text { CDS length (bp) }\end{array}$} & $\mathrm{CLC}$ & $9,289,434$ & 913,872 & $6,758,496$ & $5,921,460$ & $6,816,978$ & $23,238,501$ & $2,662,092$ & $1,107,756$ \\
\hline & IDBA_tran & $23,112,789$ & 121,116 & $9,431,703$ & $5,795,478$ & $5,143,413$ & $36,408,087$ & $2,311,569$ & $1,433,037$ \\
\hline & Trinity & $33,055,113$ & $2,749,041$ & $30,317,865$ & $14,029,893$ & $16,390,158$ & $53,633,142$ & $10,201,314$ & $4,291,554$ \\
\hline \multirow{3}{*}{$\begin{array}{l}\text { Mean extended } \\
\text { CDS length (bp) }\end{array}$} & $\mathrm{CLC}$ & 1,134 & 686 & 932 & 1,079 & 592 & 960 & 1,410 & 722 \\
\hline & IDBA_tran & 1,725 & 127 & 805 & 764 & 450 & 746 & 1,179 & 698 \\
\hline & Trinity & 1,405 & 955 & 1,048 & 1,206 & 779 & 687 & 1,305 & 739 \\
\hline
\end{tabular}

${ }^{\mathrm{a}}$ The proportion of CDSs with an extension are indicated in brackets 
Table 5 Comparisons of assembly annotatability

\begin{tabular}{|c|c|c|c|c|c|c|c|c|c|}
\hline & \multirow[b]{2}{*}{ Assembler } & \multicolumn{6}{|c|}{ Illumina derived datasets } & \multicolumn{2}{|l|}{ Simulated datasets } \\
\hline & & L. stagnalis & S. cerevisiae & Caenorhabditis sp. & D. simulans & H. rhamnoides & N. benthamiana & D. melanogaster & C. elegans \\
\hline \multirow{4}{*}{$\begin{array}{l}\text { Number of } \\
\text { uniCDS }\end{array}$} & Concatenated & 59,178 & 9,942 & 40,116 & 27,735 & 63,092 & 131,656 & 12,118 & 14,890 \\
\hline & $\mathrm{CLC}$ & 21,527 & 5,673 & 15,466 & 15,988 & 29,898 & 51,151 & 8,113 & 11,853 \\
\hline & IDBA_tran & 36,726 & 5,608 & 21,384 & 19,556 & 38,353 & 79,612 & 8,271 & 11,069 \\
\hline & Trinity & 44,545 & 9,339 & 34,356 & 23,509 & 46,571 & 88,428 & 11,351 & 12,838 \\
\hline \multirow{7}{*}{$\begin{array}{l}\text { Overall number of } \\
\text { BLASTx hits }\end{array}$} & Concatenated & 38,838 & 9,922 & 25,502 & 19,789 & 49,565 & 93,781 & 9,007 & 9,751 \\
\hline & $C L C^{\mathrm{a}}$ & 14,034 & 5,666 & 9,983 & 11,221 & 23,523 & 36,587 & 5,777 & 7,740 \\
\hline & & $(36 \%)$ & $(57 \%)$ & (39\%) & $(57 \%)$ & $(47 \%)$ & $(39 \%)$ & $(64 \%)$ & (79\%) \\
\hline & IDBA_tran ${ }^{a}$ & 23,634 & 5,598 & 13,996 & 14,107 & 30,491 & 56,665 & 6,218 & 7,489 \\
\hline & & $(61 \%)$ & $(56 \%)$ & (55\%) & $(71 \%)$ & $(62 \%)$ & $(60 \%)$ & $(69 \%)$ & (77\%) \\
\hline & Trinity $^{a}$ & 30,134 & 9,320 & 21,730 & 16,765 & 36,232 & 63,079 & 8,342 & 8,559 \\
\hline & & (78\%) & (94\%) & $(85 \%)$ & $(85 \%)$ & $(73 \%)$ & $(67 \%)$ & (93\%) & (88\%) \\
\hline \multirow{7}{*}{$\begin{array}{l}\text { Number of unique } \\
\text { BLASTx hits }\end{array}$} & Concatenated & 15,232 & 5,404 & 9,242 & 9,575 & 15,524 & 16,700 & 4,957 & 5,767 \\
\hline & $\mathrm{CLC}^{\mathrm{a}}$ & 10,958 & 5,094 & 7,492 & 8,376 & 12,405 & 12,902 & 4,664 & 5,529 \\
\hline & & $(72 \%)$ & $94 \%)$ & (81\%) & (87\%) & $(80 \%)$ & $(77 \%)$ & (94\%) & (96\%) \\
\hline & IDBA_tran ${ }^{a}$ & 12,893 & 5,223 & 8,069 & 8,868 & 13,840 & 14,900 & 4,655 & 5,302 \\
\hline & & $(85 \%)$ & (97\%) & (87\%) & (93\%) & (89\%) & (89\%) & (94\%) & (92\%) \\
\hline & Trinity $^{a}$ & 14,124 & 5,243 & 9,051 & 9,174 & 14,314 & 14,855 & 4,817 & 5,340 \\
\hline & & $(93 \%)$ & (97\%) & $(98 \%)$ & $(96 \%)$ & $(92 \%)$ & $(89 \%)$ & $(97 \%)$ & (93\%) \\
\hline
\end{tabular}

${ }^{a}$ Each value is also expressed as a percentage of the corresponding Concatenated dataset value (numbers in brackets)

Table 6 Results of BUSCO annotations

L. stagnalis S. cerevisiae Caenorhabditis sp. D. simulans H. rhamnoides N. benthamiana D. melanogaster C. elegans Illumina derived datasets

\begin{tabular}{|c|c|c|c|c|c|c|c|c|c|}
\hline \multirow{2}{*}{ BUSCO dataset } & & \multicolumn{6}{|c|}{$\underline{\text { Illumina derived datasets }}$} & \multicolumn{2}{|c|}{$\underline{\text { Simulated datasets }}$} \\
\hline & & Metazoa & Fungi & Metazoa & \multicolumn{2}{|c|}{ Arthropods } & Plants & Arthropods & Metazoa \\
\hline \multicolumn{2}{|l|}{$\begin{array}{l}\text { Number of BUSCO } \\
\text { entries }\end{array}$} & 843 & 1,438 & 843 & 2,675 & & 956 & 2,675 & 843 \\
\hline \multirow{4}{*}{$\begin{array}{l}\text { Detected BUSCO } \\
\text { entries }\end{array}$} & Concatenated & 822 & 1,357 & 720 & 2,455 & 903 & 934 & 1,204 & 442 \\
\hline & $\mathrm{CLC}$ & 779 & 1,207 & 655 & 2,159 & 843 & 811 & 1,079 & 437 \\
\hline & IDBA_tran & 813 & 1,195 & 699 & 2,242 & 881 & 903 & 1,137 & 428 \\
\hline & Trinity & 811 & 1,356 & 710 & 2,424 & 887 & 927 & 1,143 & 415 \\
\hline \multirow[t]{4}{*}{ Duplicated copies } & Concatenated & 344 & 430 & 445 & 945 & 525 & 745 & 368 & 49 \\
\hline & $\mathrm{CLC}$ & 59 & 35 & 52 & 89 & 224 & 284 & 48 & 25 \\
\hline & IDBA_tran & 210 & 70 & 189 & 662 & 361 & 569 & 149 & 28 \\
\hline & Trinity & 259 & 155 & 324 & 520 & 389 & 633 & 291 & 42 \\
\hline \multirow[t]{4}{*}{ Fragmented copies } & Concatenated & 19 & 53 & 16 & 143 & 20 & 5 & 89 & 54 \\
\hline & $\mathrm{CLC}$ & 66 & 106 & 39 & 230 & 88 & 167 & 94 & 57 \\
\hline & IDBA_tran & 20 & 59 & 16 & 174 & 33 & 63 & 93 & 54 \\
\hline & Trinity & 35 & 136 & 24 & 189 & 52 & 8 & 100 & 53 \\
\hline
\end{tabular}


elegans datasets (Table 6). Our concatenated assemblies always contained a higher number of duplicated copies than all three individual assemblers. This is apparently a weakness of our methodology that must be traded off against an assembly with more copies and fewer fragmented copies (Table 6). Our concatenated assemblies produced from the simulated datasets reflected the same patterns seen in the Illumina derived data (Table 6).

Because the NCBI databases have evolved significantly over the last two years, we downloaded the previously reported [10] cumulative transcriptome of $N$. benthamiana (http://benthgenome.qut.edu.au/), repeated the BLASTx and BUSCO searches and compared these updated results to our assembly of the same raw data. This comparison revealed that essentially the same proportion of both assemblies returned a BLASTx hit against the swiss-prot database $(75.28 \%$ versus $75.22 \%$, Table 7). Nevertheless, 250 more unique database entries were detected in our concatenated transcriptome (Table 7). These two assemblies shared 13,938 entries, while our assembly possessed 2534 unique entries and the Nakasugi et al assembly possessed 2284 unique entries (Table 7). This picture was supported by the BUSCO analysis: both assemblies shared 929 BUSCOs entries (a total of 14 BUSCOs entries were missing in both assemblies suggesting this dataset is largely complete), with five entries unique to our assembly and eight unique to the Nakasugi assembly. In addition, the number of duplicated copies was lower in our assembly than in the assembly reported by Nakasugi et al. (745 versus 785 respectively).

\section{Conclusion}

As far as we are aware this is the first study to characterize the effects of combining multiple de novo transcriptome assemblies in order to both maximize the information content, and minimize the redundancy of the resulting coding transcriptome for a variety of eukaryotes. A similar method was previously reported for transcriptomes derived from plants in order to address assembly difficulties associated with polyploidy

Table 7 Comparison of BLASTx annotation rate of both $N$. benthamiana cumulative transcriptomes

\begin{tabular}{lll}
\hline & Our study & Nakasugi et al \\
\hline Total number of transcripts & 127,526 & 234,526 \\
$\begin{array}{l}\text { Number of transcripts with } \\
\text { BLASTx hits against Swiss-prot }\end{array}$ & $95,929(75 \%)$ & $176,540(75 \%)$ \\
$\begin{array}{l}\text { Number of transcripts with a } \\
\text { unique Swiss-prot hit }\end{array}$ & 16,472 & 16,222 \\
$\begin{array}{l}\text { Number of shared transcripts } \\
\text { with a unique Swiss-prot hit }\end{array}$ & 13,938 \\
$\begin{array}{l}\text { Number unique transcripts } \\
\text { with a unique Swiss-prot hit }\end{array}$ & 2,534 & 2,284 \\
\hline
\end{tabular}

[10]. Our approach however requires only three alternative assemblies in comparison with many tens of assemblies. In general our methodology produces a more concise and information-rich coding transcriptome assembly that will make subsequent analyses more efficient; from the comparisons we conducted here on six independent eukaryotic datasets using three popular RNA-Seq assembly packages we generated on average $1.8 \mathrm{X}$ fewer transcripts, and significantly increased the degree and diversity of annotatability in comparison to any of the three individual assemblers. In addition, we tested our approach on two simulated datasets generated from reference genomes, confirming the results observed from 'real world' Illumina datasets. We believe our approach (encoded by the simple perl script provided here) will allow researchers with minimal bioinformatics experience to extract the most information from their RNA-Seq datasets. A weakness we observe in our approach is the generation of slightly more "false" transcripts and redundancy than seen in the individual assemblers we employed. This phenomenon (present in all methods used to assemble RNA-Seq data) will have an impact on subsequent analyses, for example differential gene expression (DGE). In the case of DGE analysis, this weakness can be countered to some extent by allowing multiple read mappings as implemented by Rsubread [29]. This also serves to emphasize the point that such analyses based on NGS data should always be confirmed by independent validation experiments.

\section{Additional files}

Additional file 1: Table S1. Raw NGS data characteristics pre and post quality filtering with Trimmomatic. (XLSX $10 \mathrm{~kb}$ )

Additional file 2: Parameter file for reads design with Flux simulator on D. melanogaster. (TXT 651 bytes)

Additional file 3: Perl script used to perform our assembly pipeline. (PL $43 \mathrm{~kb})$

Additional file 4: Perl script used to randomly select candidates from a list of transcripts for our PCR validation experiments. (PL $10 \mathrm{~kb}$ )

Additional file 5: Table S2. Assembly metrics of the three subassemblies and the final concatenated assembly for each of the six datasets. (XLSX $11 \mathrm{~kb})$

Additional file 6: Table S3. Clustering metrics for the three sub-assemblies for each of the six datasets. (XLSX $10 \mathrm{~kb}$ )

Additional file 7: Table S4. TransDecoder metrics for the concatenated assemblies for each of the six datasets. (XLSX $9 \mathrm{~kb}$ )

Additional file 8: Table S5. Effect of the CD-HIT-EST aS value on clustering. (XLSX 13 kb)

Additional file 9: Table S6. $p$-values generated by Kolmogrov-Smirnov distribution tests. (XLSX 9 kb)

Abbreviations

BUSCO: Benchmarking Universal Single Copy Orthologs; CDS: Coding DNA sequence; DGE: Differential gene expression; DNA: DeoxyriboNucleic Acid; mRNA: messenger RNA; NGS: Next generation sequencing; ORFs: Open 
reading frames; PCR: Polymerase chain reaction; RNA: RiboNucleic Acid; SRA: Sequence read archive

\section{Acknowledgements}

We gratefully acknowledge the developers of BUSCO for early access to the plant database and two anonymous reviewers that helped to improve the manuscript.

\section{Funding}

This work was supported by a Deutsche Forschungsgemeinschaft grant to DJJ (JA 2108/1-2)

\section{Availability of data and materials}

All data used to perform the analyes described in this work are freely available. The raw RNA-seq data for Drosophila simulans, Caenorhabditis sp., Saccharomyces cerevisiae, Hippophae rhamnoides and Nicotiana benthamiana were obtained from the NCBI sequence read archive (SRA) (respective accession numbers: SRR1956911, ERR690851, SRR1924287, SRP011938 and SRA066161 (single end data omitted). The newly generated L. stagnalis data is available under SRR3469744. The Perl scripts described are available as Additional files 3 and 4

\section{Authors' contributions}

Both authors conceived, designed and refined the study. NC carried out all molecular work and wrote and refined the Perl scripts. Both authors wrote and drafted the manuscript. Both authors read and approved the final manuscript.

\section{Competing interests}

The authors declare that they have no competing interests.

\section{Consent for publication}

Not applicable.

\section{Ethics approval and consent to participate}

All experiments described here comply with the current laws and regulations of the country they were conducted in.

\section{Received: 7 September 2016 Accepted: 1 December 2016}

\section{Published online: 09 December 2016}

\section{References}

1. Ozsolak F, Milos PM. RNA sequencing: advances, challenges and opportunities. Nat Rev Genet. 2011;12:87-98.

2. O'Neil ST, Dzurisin JDK, Carmichael RD, Lobo NF, Emrich SJ, Hellmann J. Population-level transcriptome sequencing of nonmodel organisms Erynnis propertius and Papilio zelicaon. BMC Genomics. 2010;11:310.

3. Jänes J, Hu F, Lewin A, Turro E. A comparative study of RNA-seq analysis strategies. Brief Bioinform. 2015;16:932-40.

4. Metzker ML. Sequencing technologies - the next generation. Nat Rev Genet. 2010;11:31-46

5. Martin JA, Wang Z. Next-generation transcriptome assembly. Nat Rev Genet. 2011:12:671-82

6. Ghangal R, Chaudhary S, Jain M, Purty RS, Chand SP. Optimization of de novo short read assembly of seabuckthorn (Hippophae rhamnoides L) transcriptome. PLoS ONE. 2013;8:e72516.

7. Posnien N, Zeng V, Schwager EE, Pechmann M, Hilbrant M, Keefe JD, et al A comprehensive reference transcriptome resource for the common house spider Parasteatoda tepidariorum. PLoS ONE. 2014:9:e104885.

8. Finseth FR, Harrison RG. A comparison of next generation sequencing technologies for transcriptome assembly and utility for RNA-Seq in a nonmodel bird. PLOS ONE. 2014;9:e108550.

9. Chang Z, Li G, Liu J, Zhang Y, Ashby C, Liu D, et al. Bridger: a new framework for de novo transcriptome assembly using RNA-seq data. Genome Biol. 2015;16:30.

10. Nakasugi K, Crowhurst R, Bally J, Waterhouse P. Combining transcriptome assemblies from multiple de novo assemblers in the allo-tetraploid plant Nicotiana benthamiana. PLoS ONE. 2014;9:e91776.

11. Duan J, Xia C, Zhao G, Jia J, Kong X. Optimizing de novo common wheat transcriptome assembly using short-read RNA-Seg data. BMC Genomics. 2012;13:392
12. Zhao Q-Y, Wang Y, Kong Y-M, Luo D, Li X, Hao P. Optimizing de novo transcriptome assembly from short-read RNA-Seq data: a comparative study. BMC Bioinform. 2011;12 Suppl 14:S2.

13. O'Neil ST, Emrich SJ. Assessing De Novo transcriptome assembly metrics for consistency and utility. BMC Genomics. 2013;14:465.

14. Simão FA, Waterhouse RM, loannidis P, Kriventseva EV, Zdobnov EM. BUSCO: assessing genome assembly and annotation completeness with single-copy orthologs. Bioinformatics. 2015;31:3210-2.

15. Grabherr MG, Haas BJ, Yassour M, Levin JZ, Thompson DA, Amit I, et al. Full-length transcriptome assembly from RNA-Seq data without a reference genome. Nat Biotechnol. 2011;29:644-52.

16. Peng $Y$, Leung HCM, Yiu S-M, Lv M-J, Zhu X-G, Chin FYL. IDBA-tran: a more robust de novo de Bruijn graph assembler for transcriptomes with uneven expression levels. Bioinformatics. 2013;29:326-34.

17. Mann K, Jackson DJ. Characterization of the pigmented shell-forming proteome of the common grove snail Cepaea nemoralis. BMC Genomics. 2014;15:249.

18. Bolger AM, Lohse M, Usadel B. Trimmomatic: a flexible trimmer for Illumina sequence data. Bioinformatics. 2014;30:2114-20.

19. Li B, Dewey CN. RSEM: accurate transcript quantification from RNA-Seq data with or without a reference genome. BMC Bioinform. 2011;12:323.

20. Griebel T, Zacher B, Ribeca P, Raineri E, Lacroix V, Guigó R, et al. Modelling and simulating generic RNA-Seq experiments with the Flux simulator. Nucleic Acids Res. 2012:40:10073-83.

21. Fu L, Niu B, Zhu Z, Wu S, Li W. CD-HIT: accelerated for clustering the nextgeneration sequencing data. Bioinformatics. 2012;28:3150-2.

22. Haas BJ, Papanicolaou A, Yassour M, Grabherr M, Blood PD, Bowden J, et al. De novo transcript sequence reconstruction from RNA-seq using the Trinity platform for reference generation and analysis. Nat Protoc. 2013;8:1494-512.

23. Untergasser A, Cutcutache I, Koressaar T, Ye J, Faircloth BC, Remm M et al. Primer3-new capabilities and interfaces. Nucleic Acids Res. 2012:40:e115.

24. Feldmeyer B, Wheat CW, Krezdorn N, Rotter B, Pfenninger M. Short read Illumina data for the de novo assembly of a non-model snail species transcriptome (Radix balthica, Basommatophora, Pulmonata), and a comparison of assembler performance. BMC Genomics. 2011:12:317.

25. Li B, Fillmore N, Bai Y, Collins M, Thomson JA, Stewart R, et al. Evaluation of de novo transcriptome assemblies from RNA-Seq data. Genome Biol. 2014; 15:553

26. Amin S, Prentis PJ, Gilding EK, Pavasovic A. Assembly and annotation of a non-model gastropod (Nerita melanotragus) transcriptome: a comparison of de novo assemblers. BMC Res Notes. 2014;7:488

27. Belancio VP, Hedges DJ, Deininger P. Mammalian non-LTR retrotransposons: For better or worse, in sickness and in health. Genome Res. 2008;18:343-58.

28. Blumenthal T. Operons in eukaryotes. Brief Funct Genomic Proteomic. 2004;3:199-211

29. Liao Y, Smyth GK, Shi W. The Subread aligner: fast, accurate and scalable read mapping by seed-and-vote. Nucleic Acids Res. 2013;41:e108.

\section{Submit your next manuscript to BioMed Central and we will help you at every step:}

- We accept pre-submission inquiries

- Our selector tool helps you to find the most relevant journal

- We provide round the clock customer support

- Convenient online submission

- Thorough peer review

- Inclusion in PubMed and all major indexing services

- Maximum visibility for your research

Submit your manuscript at www.biomedcentral.com/submit
Biomed Central 